\title{
Ruthenium-106 Brachytherapy with or without Additional Local Therapy Shows Favorable Outcome for Variable-Sized Choroidal Melanomas in Korean Patients
}

\author{
Yeona Cho, $\mathrm{MD}^{1}$ \\ Jee Suk Chang, MD ${ }^{1}$ \\ Jin Sook Yoon, MD, PhD2 \\ Sung Chul Lee, MD, PhD'2 \\ Yong Bae Kim, MD, PhD' \\ Joo Ho Kim, CMD, RTT ${ }^{1}$ \\ Ki Chang Keum, MD, $\mathrm{PhD}^{1}$
}

${ }^{1}$ Department of Radiation Oncology, Yonsei Cancer Center, Yonsei University College of Medicine, Seoul, ${ }^{2}$ Department of Ophthalmology, Institute of Vision Research, Severance Hospital, Yonsei University College of Medicine, Seoul, Korea
Correspondence: Ki Chang Keum, MD, PhD Department of Radiation Oncology,

Yonsei Cancer Center, Yonsei University College of Medicine, 50 Yonsei-ro, Seodaemun-gu,

Seoul 03722, Korea

Tel: 82-2-2227-7823

Fax: 82-2-2228-8112

E-mail: kckeum@yuhs.ac

Received August 18, 2016

Accepted March 3, 2017

Published Online March 24, 2017

\section{Purpose}

The purpose of this study was to report clinical outcomes of ruthenium-106 $\left({ }^{106} \mathrm{Ru}\right)$ brachytherapy with or without additional local therapy for choroidal melanomas in Korean patients.

\section{Materials and Methods}

A total of 88 patients diagnosed with choroidal melanomas were treated with ${ }^{106} \mathrm{Ru}$ brachytherapy between 2006 and 2012. Patients were divided into two groups according to their tumor height: a large group ( $\geq 6 \mathrm{~mm}, \mathrm{n}=50$ ) and a small group ( $<6 \mathrm{~mm}, \mathrm{n}=38$ ). Most patients in the large group received combined therapy with local excision and/or transpupillary thermotherapy. In general, 85-95 Gy was administered to the apex of the tumor, while 100 Gy was administered to the point 2-6 $\mathrm{mm}$ from the outer surface of the sclera for patients undergoing combined therapy.

\section{Results}

The median follow-up duration was 30 months. The 3-year local control rate was significantly higher in the small group than in the large group ( $94 \%$ vs. $70 \%, p=0.047$ ). The free from distant metastasis (FFDM) rate and the overall survival (OS) rate were also higher in patients in the small group (3-year FFDM, 97\% vs. 76\%; $p=0.031$ and 3 -year OS, 97\% vs. $72 \%$; $p=0.036)$. A total of 13 patients underwent enucleation. The eye-preservation rate was also higher in the small group (3-year eye-preservation rate, $94 \%$ vs. $70 \% ; p=0.050$ ), and tumor height was a significant prognostic factor for eye-preservation.

\section{Conclusion}

${ }^{106} \mathrm{Ru}$ brachytherapy showed favorable outcomes in small choroidal melanomas in Korean patients. Although additional local treatment could improve eye-preservation rate for large tumors, other strategies should be considered for disease control.

\section{Introduction}

Choroidal melanomas are the most common primary intraocular malignancies in adults. However, choroidal melanoma predominantly affects Caucasians, while it is very rare in Asian populations. Despite the low incidence rates in Asian populations, the tumors usually affect younger patients, tend to be larger, and present a high number of epitheloid cell types, resulting in a poorer prognosis in Asian
Key words

Uveal melanoma, Brachytherapy, Ruthenium radioisotopes patients than in Caucasians [1].

Until the introduction of plaque brachytherapy using iodine $125\left({ }^{125} \mathrm{I}\right)$ in the $1960 \mathrm{~s}$, enucleation was the standard treatment for patients with choroidal melanomas. Although the most important treatment goal is survival free of disease recurrence, both patients and clinicians focus on preservation of function and cosmetic appearance as well. The Collaborative Ocular Melanoma Study confirmed the efficacy of plaque brachytherapy in a multicenter randomized trial including 1,317 patients, which did not reveal any inferiority of 


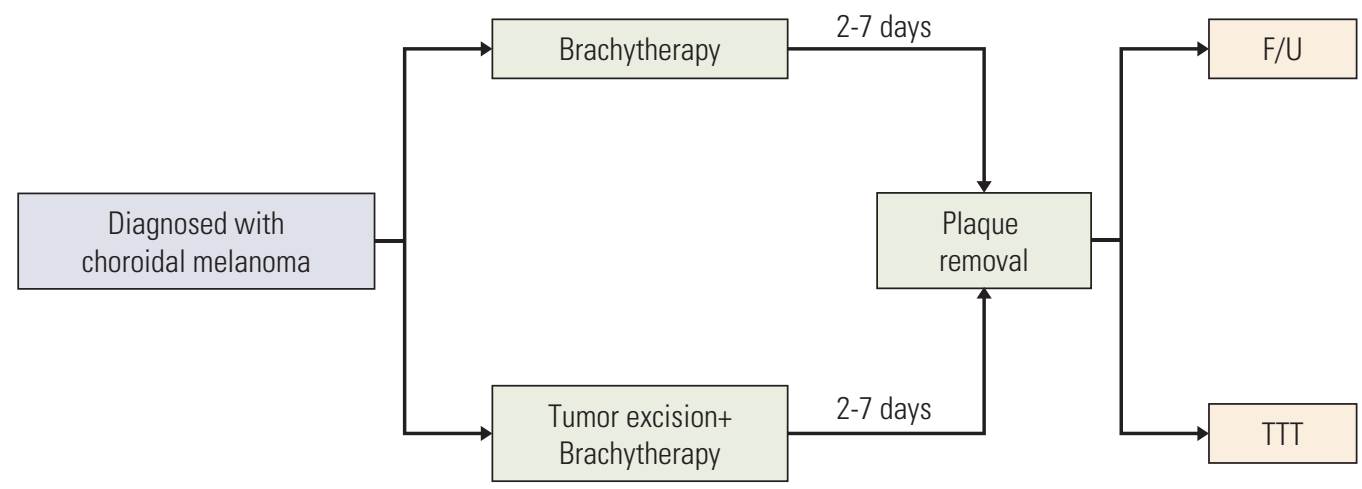

Fig. 1. Treatment flow diagram. F/U, follow-up; TTT, transpupillary thermotherapy.

${ }^{125}$ I brachytherapy to enucleation, showing comparable 5 -year overall survival (OS) (81\% vs. 82\%) and 5-year cancerspecific survival $(89 \%$ vs. $91 \%)$ rates [2,3]. Moreover, ${ }^{125}$ I brachytherapy did pose any additional risk of loss of visual acuity in the fellow eye for at least 10 years after treatment for choroidal melanoma [4]. Today, plaque brachytherapy is a major modality for the management of choroidal melanomas because it is considered a reasonable eye-preserving alternative to enucleation. Although the benefits of plaque brachytherapy may be reduced by impaired vision resulting from radiation toxicity, this treatment modality has major functional and cosmetic advantages.

Since its introduction, ruthenium-106 $\left({ }^{106} \mathrm{Ru}\right)$ brachytherapy has been increasingly used for the treatment of small- to medium-sized choroidal melanomas [5-7]. A ${ }^{106} \mathrm{Ru}$ plaque providing 1,000 Gy to the scleral surface conveys less than $100 \mathrm{~Gy}$ to a distance over $7 \mathrm{~mm}$ from the base, giving an insufficient radiation dose to the apex of large tumors $[8,9]$. Insufficient radiation to the apex could reduce the local tumor control rate and increase the risk of recurrence, as well as metastases and mortality. Several studies have reported outcomes of the combination of the brachytherapy and transpupillary thermotherapy (TTT) $[10,11]$. This combination therapy was introduced to enable eye-preservation for patients with tumors larger than $5 \mathrm{~mm}$, although it has also been used to lessen the radiation dose for smaller tumors to reduce the risk of radiation-related toxicities. Moreover, patients with insufficient tumor regression after brachytherapy or with recurrent tumors can be re-treated with TTT.

Before the introduction of ${ }^{106} \mathrm{Ru}$ plaques (Eckert \& Ziegler BEBIG, Berlin, Germany) to our institution in October 2006, Gamma knife surgery (GKS) using a Leksell $\gamma$-knife (Elekta Instruments AB, Stockholm, Sweden) was the only eye-sparing treatment for choroidal melanomas available in Korea. Since 2006, we have primarily performed brachytherapy with additional local treatment, including TTT and local excision, as eye-preserving therapy for choroidal melanomas. This study was conducted to report the early clinical outcomes of this eye-preserving treatment strategy for variablesized choroidal melanomas in Korean patients.

\section{Materials and Methods}

\section{Patient selection}

We identified 93 consecutive patients who were diagnosed with uveal malignant melanomas and treated with ${ }^{106} \mathrm{Ru}$ brachytherapy between 2006 and 2012 in our institution. Patients with iris and ciliary body melanomas were excluded $(n=5)$ from this study. Because ${ }^{106} \mathrm{Ru}$ plaque therapy is generally considered to treat tumors less than 6-7 $\mathrm{mm}$ in height, our patients were divided into a large group (tumor height $\geq 6 \mathrm{~mm}$ ) and a small group (tumor height $<6 \mathrm{~mm}$ ) according to their tumor height, as measured by ultrasonography.

\section{Treatment methods}

The treatment flow diagram is presented in Fig. 1. The treatment was intended to deliver 85-95 Gy to the apex of the tumor. However, owing to rapid radiation dose fall-off of ${ }^{106} \mathrm{Ru}, 80 \%$ of the dose had already been absorbed at $5 \mathrm{~mm}$ from the applicator. Thus, the dose to the apex is generally considered insufficient for treatment of large tumors. The patients were informed of this limitation, but some patients preferred brachytherapy anyway in an effort to preserve their eyes.

The three most frequently used applicators have diameters 
of $15.3,17.9$, and $20.2 \mathrm{~mm}$ with active diameters of $13.5,15.8$, and $18 \mathrm{~mm}$, respectively. Applicators for the treatment of juxtapapillary tumors (those located immediately adjacent to the optic nerve) have a section cutout for the optic nerve.

Some patients treated with brachytherapy received additional TTT and / or local excision. Local excision of the tumor was conducted before brachytherapy on the same day as the brachytherapy, which was delivered as an adjuvant treatment. Planned TTT was performed with an adjuvant goal after brachytherapy in patients who did not undergo local excision. Unplanned TTT was performed if tumor regression during follow-up was not sufficient after brachytherapy, regardless of initial tumor size. The first planned TTT procedure was performed at the time of plaque removal using an infrared diode laser and a slit-lamp delivery system. During the follow-up period, additional TTT procedures were performed in patients in an outpatient setting at intervals of 3 months.

Enucleation was generally recommended for patients with large tumors (height $\geq 6 \mathrm{~mm}$ ) because ${ }^{106} \mathrm{Ru}$ brachytherapy is not suitable for such tumors. However, for patients who refused enucleation, local excision of the tumor was performed to reduce the height of the apex. If the tumor was mainly located near the ciliary body, a transscleral tumor resection was performed, while if the tumor had a posterior location and a basal diameter of less than $15 \mathrm{~mm}$, endoresection with pars plana vitrectomy was performed.

\section{Evaluation of outcomes}

The primary end-point was the eye-preservation rate (free from enucleation). In addition, local control, $\mathrm{OS}$ and the rate of distant metastasis were assessed. Survival was calculated from the date of the insertion of the eye plaque to any event. Patients lost to follow-up were censored. Late toxicity was evaluated according to the Radiation Therapy Oncology Group scale.

Tumor response was evaluated with fundoscopy and ultrasonography, with a flat scar or a regressed lesion not showing any signs of tumor activity representing tumor control. Local recurrence was defined as signs of tumor activity on fluorescein angiography, or documented tumor growth of more than $20 \%$ of the initial diameter or thickness (Response Evaluation Criteria in Solid Tumors criteria). Metastases were detected through routine follow-up investigations (liver ultrasonography and blood chemistry tests) or using ${ }^{18} \mathrm{~F}$-fluorodeoxyglucose positron emission tomography.

To assess the toxicity of treatment, regular follow-ups for visual acuity and other ophthalmologic evaluations were performed, in which complications such as retinopathy, maculopathy, optic neuropathy, retinal hemorrhage, and exuda- tive retinal detachment were evaluated. In this study, only issues that needed further treatment were defined as complications. Vision was assessed using the World Health Organization (WHO) criteria, with low vision defined as a visual acuity less than $1 / 3$ and a vision $<0.1$ considered legal blindness.

\section{Statistical analysis}

The survival curves were estimated using the KaplanMeier method, and comparisons between the two patients groups were made with the log-rank test. Significant variables in univariate analysis $(\mathrm{p}<0.05)$ were entered into a multivariable Cox regression model to identify independent predictors of eye-preservation rate, distant metastasis, and OS. $\mathrm{p}<0.05$ were considered significant. Statistical analyses were performed using SPSS ver. 20.0.0 (SPSS Inc., Chicago, IL).

\section{Results}

\section{Patient and treatment characteristics}

Between 2006 and 2012, 93 patients with choroidal melanomas were treated with ${ }^{106} \mathrm{Ru}$ brachytherapy. Five patients with iris and ciliary body tumors were excluded, leaving 88 patients in the analysis. The median follow-up period was 30 months (range, 2 to 79 months). The patients and treatment characteristics are summarized in Table 1 . The median age was 49 years (range, 19 to 82 years). The initial visual acuity in the affected eye was $\geq 0.10$ in $71 \%(n=63)$ and $\geq 0.9$ in $16 \%(n=14)$ of patients. Twenty-five (29\%) patients were diagnosed with legal blindness $(<0.1)$ prior to treatment and 10 patients $(11 \%)$ had visual impairment ( $<20 / 60-20 / 200$, WHO definition). The median basal tumor diameter was $11.4 \mathrm{~mm}$ (range, 2.6 to $17.3 \mathrm{~mm}$ ), and the median tumor height was $6.8 \mathrm{~mm}$ (range, 1.7 to $13.7 \mathrm{~mm}$ ). The number of patients with a tumor height $\geq 6 \mathrm{~mm}$ (large group) was $50(57 \%)$, and there were $38(43 \%)$ patients in the small group.

Combined therapy was used in the treatment of $75 \%(n=65)$ of the patients. In the large group (tumor height $\geq 6 \mathrm{~mm}$ ), $96 \%$ $(n=48)$ of the patients received combined local therapy, while in the small group, $44.7 \%(\mathrm{n}=17)$ of the patients were treated with combined treatment. The radiation dose was delivered to $2-6 \mathrm{~mm}$ from the outer surface of the sclera for $47.7 \%(\mathrm{n}=42)$ of the entire sample, based on the extent of local excision and use of TTT. For some of the patients who were treated with TTT alone as an additional local therapy and for all patients who did not receive additional treatment, the tumor apex was 
Table 1. Patient and treatment characteristics

\begin{tabular}{|c|c|c|c|}
\hline Characteristic & Total $(n=88)$ & Small group $(n=38)$ & Large group $(n=50)$ \\
\hline \multicolumn{4}{|l|}{ Sex } \\
\hline Male & $44(50.0)$ & $17(44.7)$ & $27(54.0)$ \\
\hline Female & $44(50.0)$ & $21(55.3)$ & $23(46.0)$ \\
\hline \multicolumn{4}{|l|}{ Age (yr) } \\
\hline$<50$ & $44(50.0)$ & $14(36.8)$ & $30(60.0)$ \\
\hline$\geq 50$ & $44(50.0)$ & $24(63.2)$ & $20(40.0)$ \\
\hline \multicolumn{4}{|l|}{ Initial visual acuity } \\
\hline$<0.1$ (legal blindness) & $25(28.4)$ & $9(23.7)$ & $16(32.0)$ \\
\hline $0.1-0.8$ & $49(55.7)$ & $24(63.2)$ & $25(50.0)$ \\
\hline$\geq 0.9$ & $14(15.9)$ & $5(13.2)$ & $9(18.0)$ \\
\hline \multicolumn{4}{|l|}{ Pigmentation } \\
\hline Melanotic & $75(85.2)$ & $32(84.2)$ & $43(86.0)$ \\
\hline Amelanotic & $13(14.8)$ & $6(15.8)$ & $7(14.0)$ \\
\hline \multicolumn{4}{|l|}{ Proximity to optic nerve } \\
\hline Central/Juxtapapillary & $32(36.4)$ & $15(39.5)$ & $17(34.0)$ \\
\hline Peripheral & $56(63.6)$ & $23(60.5)$ & $33(66.0)$ \\
\hline Basal diameter (mm) & $11.4(2.6-17.3)$ & $9.8(4.0-15.2)$ & $12.5(3.0-17.3)$ \\
\hline Tumor height (mm) & $6.8(1.7-13.7)$ & $4.7(1.7-6)$ & $8.2(6.3-13.7)$ \\
\hline \multicolumn{4}{|l|}{ COMS stage } \\
\hline Small & $4(4.5)$ & $3(7.9)$ & $1(2.0)$ \\
\hline Medium & $73(83.0)$ & $35(92.1)$ & $38(76.0)$ \\
\hline Large & $11(12.5)$ & 0 & $11(22.0)$ \\
\hline \multicolumn{4}{|l|}{ Visual acuity } \\
\hline$<0.1$ & $25(28.4)$ & $9(23.7)$ & $16(32.0)$ \\
\hline $0.1-0.5$ & $28(31.8)$ & $13(34.2)$ & $15(30.0)$ \\
\hline $0.6-0.8$ & $21(23.9)$ & $11(28.9)$ & $10(20.0)$ \\
\hline$\geq 0.9$ & $14(15.9)$ & $5(13.2)$ & $9(18.0)$ \\
\hline \multicolumn{4}{|l|}{ Combined treatment } \\
\hline No & $23(26.1)$ & $21(55.3)$ & $2(4.0)$ \\
\hline Yes & $65(73.9)$ & $17(44.7)$ & $48(96.0)$ \\
\hline TTT alone & $39(44.3)$ & $15(39.5)$ & $24(48.0)$ \\
\hline Tumor excision alone & $22(25.0)$ & $1(2.6)$ & $21(42.0)$ \\
\hline Both & $4(4.5)$ & $1(2.6)$ & $3(6.0)$ \\
\hline \multicolumn{4}{|l|}{ Prescription point } \\
\hline Apex & $46(52.3)$ & $36(94.7)$ & $10(20.0)$ \\
\hline 2-6 mm from the sclera & $42(47.7)$ & $2(5.3)$ & $40(80.0)$ \\
\hline \multicolumn{4}{|l|}{ Dose to sclera (Gy) } \\
\hline$<500$ & $42(47.7)$ & $22(57.9)$ & $24(48.0)$ \\
\hline $500-1,000$ & $39(44.3)$ & $16(42.1)$ & $19(38.0)$ \\
\hline$>1,000$ & $7(8.0)$ & 0 & $7(14.0)$ \\
\hline Dose rate to sclera (cGy/hr) & $578.5(85.2-1,058)$ & $486.1(174.2-825.8)$ & $800.2(76.98-1,385)$ \\
\hline Time to removal (hr) & $85(12-238)$ & 85 (19.3-184) & $96(51-238)$ \\
\hline
\end{tabular}

Values are presented as number $(\%)$ or median (range). COMS, Collaborative Ocular Melanoma Study; TTT, transpulpillary thermotherapy.

the prescription point.

Some patients $(n=15,39.5 \%)$ in the small group also received additional TTT when tumor regression was deemed insufficient after brachytherapy, while two patients received local excision before brachytherapy. One patient experienced rupture of the tumor during vitrectomy and eventually received internal choroidectomy. The other patient, who had a tumor with a size of $12.5 \mathrm{~mm}$ (basal diameter) $\times 5.9 \mathrm{~mm}$ (tumor height), 


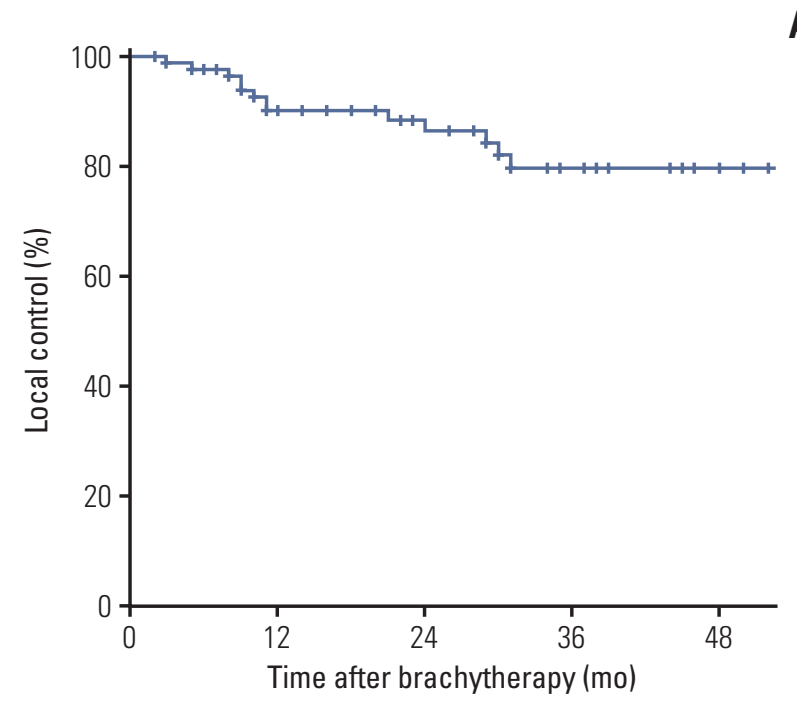

A

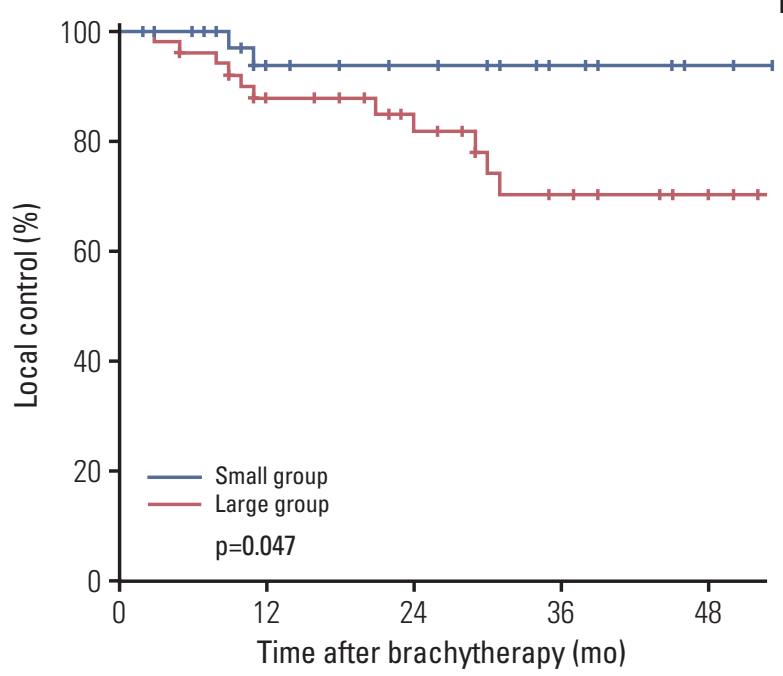

Fig. 2. Local control of all patients (A) and two groups (B).

A
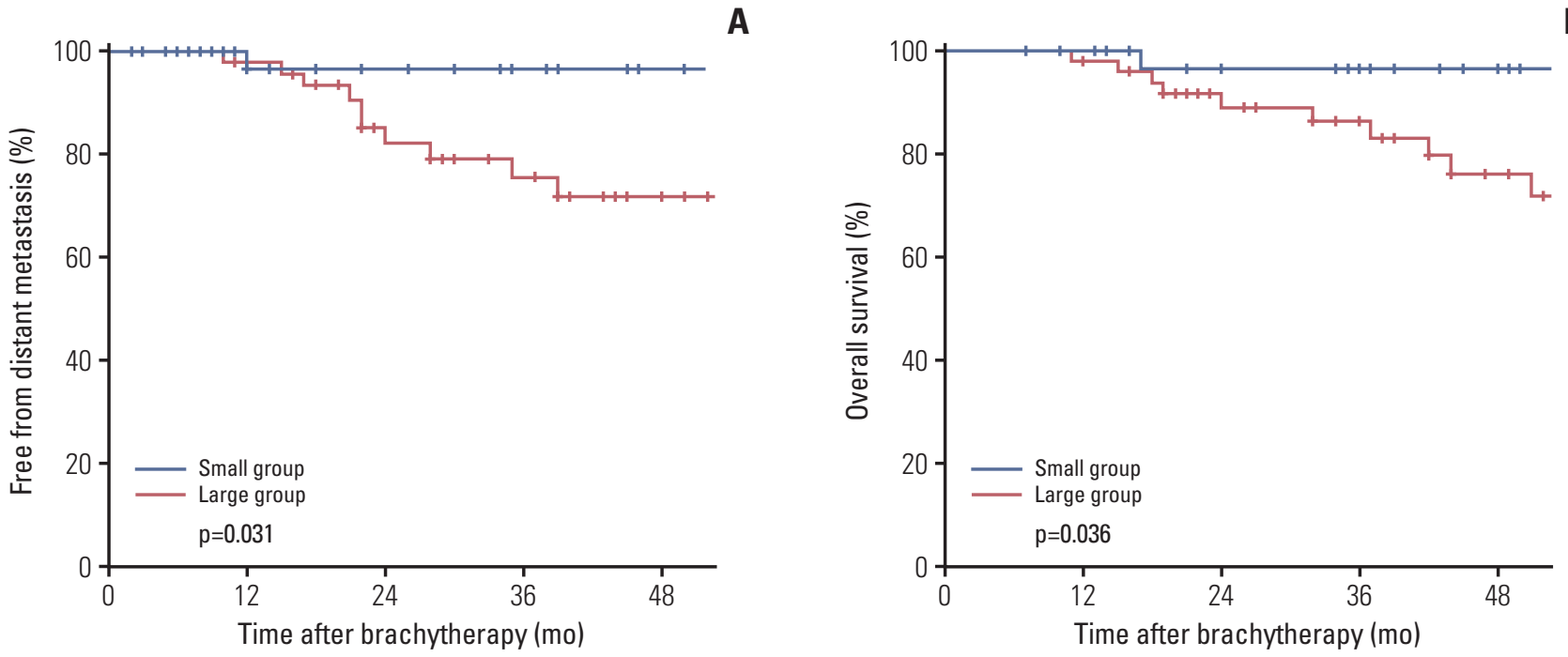

Fig. 3. Free from distant metastasis (A) and overall survival (B) curves of two groups.

was included in the small group in this study. However, he received local excision based on the decision of his physicians.

\section{Local control, distant metastasis, and OS}

Local progression was diagnosed in 13 patients. The 3-year local control rate was $80 \%$ (Fig. 2), and the 3-year local control rate was significantly higher in the small group than in the large group ( $94 \%$ vs. $70 \%$, respectively; $\mathrm{p}=0.047$ ). Salvage treatments for local recurrence consisted of enucleation $(n=12)$ and additional TTT $(\mathrm{n}=1)$.

Distant metastases were diagnosed in 12 patients, and one patient died. The 3-year free from distant metastasis (FFDM) rate was $84 \%$, and the OS was $90 \%$ for all patients. The FFDM 
A
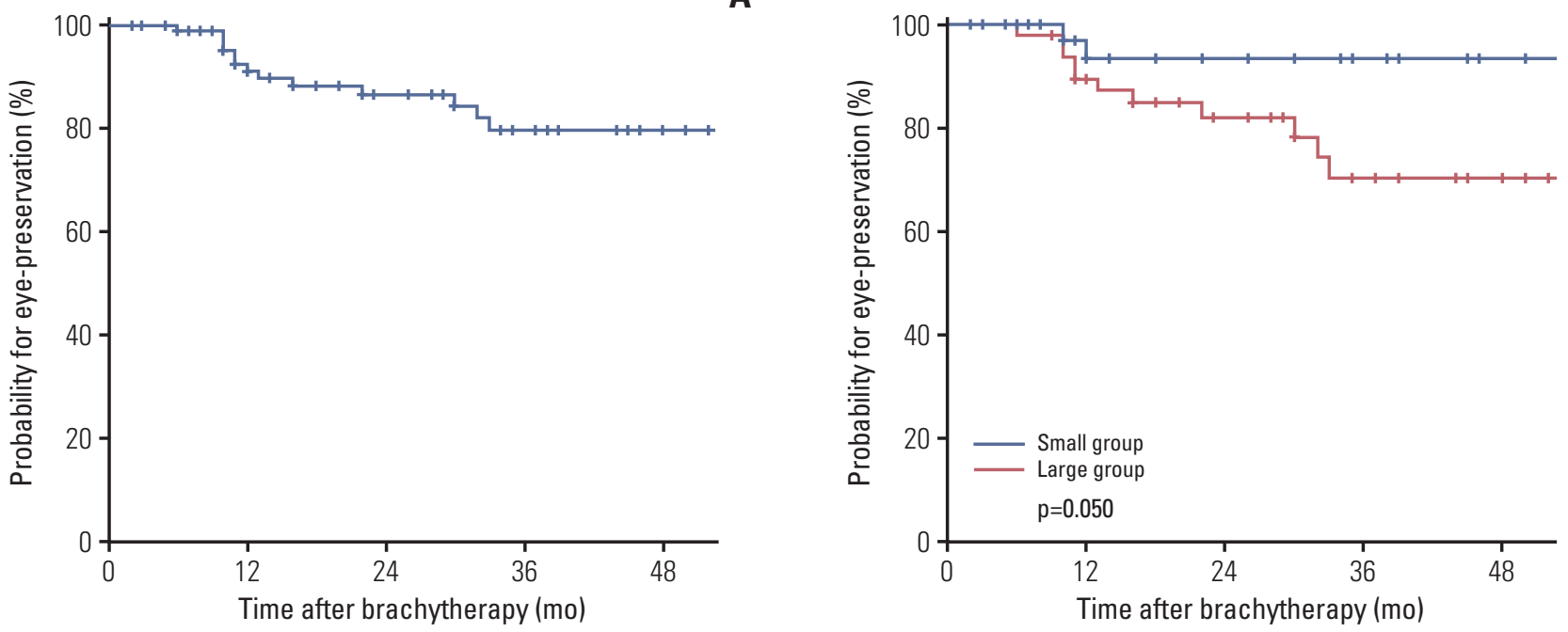

Fig. 4. Eye-preservation rate of all patients (A) and two groups (B).

Table 2. Radiation related toxicities

\begin{tabular}{lcccl} 
Radiation related toxicity & No. of events (\%) & RTOG grade & Interval (mo) & \multicolumn{1}{c}{ Treatment } \\
Glaucoma & $3(3)$ & 2 & $7,14,15$ & Seton operation, medical treatment \\
Vitreous hemorrhage & $2(2)$ & & 12,14 & Trans pars plana vitrectomy \\
Retinal detachment & $1(1)$ & 3 & 20 & Endolaser photocoagulation \\
Phthisis & $1(1)$ & 3 & 10 & Enucleation \\
Total & $6(7)$ & & \\
\hline
\end{tabular}

RTOG, Radiation Treatment Oncology Group.

and OS rates were also significantly higher in patients in the small group (3-year FFDM, 97\% vs. 76\%; $\mathrm{p}=0.031$ and 3-year OS, $97 \%$ vs. $72 \%$; $\mathrm{p}=0.036$ ) (Fig. 3).

\section{Eye preservation}

Among the 88 patients, 13 eventually underwent enucleation (12 patients for local recurrence and one patient for a complication), resulting in a 3-year actuarial eye-preservation rate of $80 \%$. In the small group, only two patients underwent enucleation because of tumor progression, giving a 3-year eyepreservation rate of $94 \%$. The eye-preservation rate was significantly higher in this group when compared with the large group (3-year eye-preservation rate, 94\% vs. 70\%, respectively; $\mathrm{p}=0.050)$ (Fig. 4).

\section{Toxicities}

The total rate of complications requiring further treatment was only $7 \%(n=6)$. The types, number of cases, and interval from brachytherapy are shown in Table 2 . Two patients with glaucoma received medical treatment (mannitol, avastin), and one of them eventually received enucleation due to tumor progression, while the other underwent the Seton operation. Other complications, including vitreous hemorrhage and retinal detachment, were tolerable with proper intervention. One patient in the large group who had a tumor with a height of 9 $\mathrm{mm}$ and received TTT after brachytherapy wanted to undergo enucleation for phthisis 10 months after brachytherapy. The dose to the sclera of this patient exceeded 1,000 Gy.

Among the 63 patients with a pre-treatment visual acuity greater than $0.10,39(62 \%)$ showed a deterioration of visual acuity in the treated eye to $<0.10$, which was considered to be legal blindness. The 3-year functional eye-preservation rate 


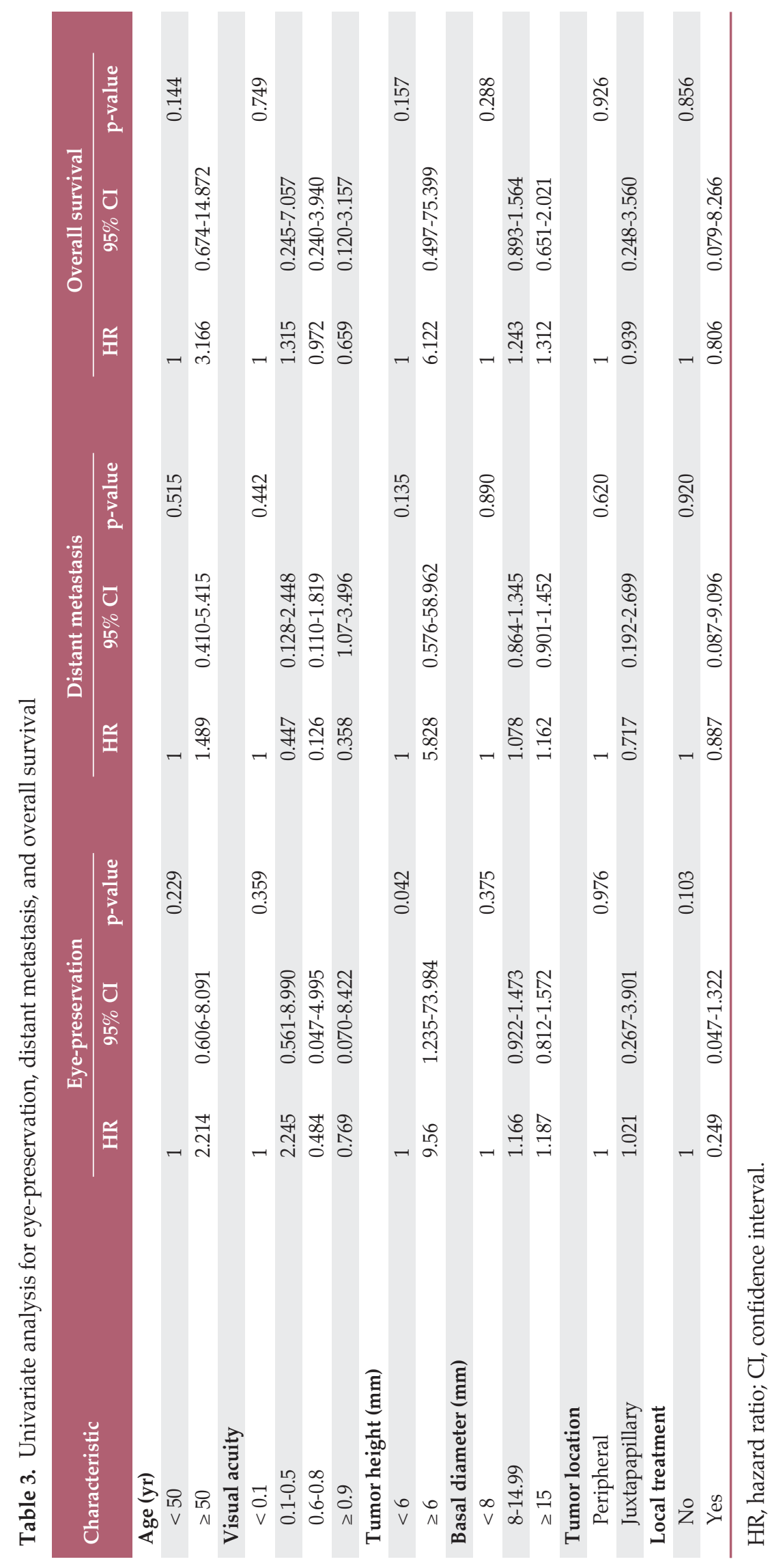


was $58 \%$, and $50 \%$ of tumors were close to the macula or optic disc in these patients.

\section{Prognostic factors}

Cox regression analysis was used to evaluate prognostic factors for eye-preservation, distant metastasis, and OS (Table 3 ). The following potential prognostic variables were examined in the Cox proportional hazards models: age $(<50$ years vs. $\geq 50$ years), initial visual acuity, tumor height ( $<6 \mathrm{~mm}$ vs. $\geq 6$ $\mathrm{mm}$ ), basal diameter, tumor location, and local treatment. The significant prognostic factor for eye-preservation was tumor height $\geq 6 \mathrm{~mm}$ (hazard ratio, 9.560; 95\% confidence interval, 1.235 to $73.984, \mathrm{p}=0.042$ ), while it was not significantly associated with distant metastasis or OS $(\mathrm{p}=0.135$ and $\mathrm{p}=0.157$, respectively). Other characteristics including age, initial visual acuity, basal diameter, and tumor location did not affect the patients' prognosis.

\section{Discussion}

In this study, we showed favorable local tumor control and eye-preservation rates, especially in patients in the small group (3-year local control, 94\%; eye-preservation rate, 94\%). These results are comparable to those reported by other groups for small or medium-sized tumors [12].

Although enucleation has been the most common primary treatment for large choroidal melanomas, previous studies showed that ${ }^{125}$ I brachytherapy could be a possible alternative treatment with regard to survival and local tumor control $[13,14]$. However, few studies have investigated the use of beta-radiation emitting ${ }^{106} \mathrm{Ru}$ to treat large choroidal melanomas. ${ }^{106} \mathrm{Ru}$ may not be effective because of its limited depth of penetration, which could prevent it from reaching the apex of thick tumors [15]. When compared with ${ }^{125}$ I, ${ }^{106} \mathrm{Ru}$ has a threefold faster radiation dose fall-off rate. The effective amount of radiation of 80-100 Gy reaches less than $7 \mathrm{~mm}$ when a ${ }^{106} \mathrm{Ru}$ eye plaque with 1,000 Gy to the outer surface of sclera is applied. Thus, a thicker tumor might receive an insufficient radiation dose to its apex. On the other hand, when the same radiation dose is delivered to the apex, the base receives up to three times more radiation with ${ }^{106} \mathrm{Ru}$ than with ${ }^{125}$ I, which might cause extensive damage to the sclera. Based on these findings, most patients in the large group $(96 \%)$ received additional local treatment with TTT, tumor excision or both.

Tumor necrosis caused by TTT is induced to a depth of 3 $\mathrm{mm}$ from the apex [16]. The effectiveness of combined use of ${ }^{106} \mathrm{Ru}$ brachytherapy and TTT has been demonstrated in sev- eral studies. As with TTT, the apex of the tumor is treated up to $3 \mathrm{~mm}$, while with brachytherapy, the highest dose is administered to the tumor base $[10,17,18]$. In addition, among patients in the large group (height $\geq 6 \mathrm{~mm}$ ), local excision of the tumor was performed in $46 \%$ of patients. In such cases, the prescription point was determined to be $2 \mathrm{~mm}$ from the outer surface of the sclera. Augsburger et al. [19] previously reported treatment of choroidal melanomas with extrascleral extension by surgical excision of the extrascleral nodule followed immediately by plaque radiotherapy of the intraocular tumor. They demonstrated the efficacy of a combination of local excision and brachytherapy for the treatment of choroidal melanomas showing that brachytherapy alone was not sufficient. Adding these local treatments to ${ }^{106} \mathrm{Ru}$ brachytherapy could increase local control in patients with tumor heights exceeding $6 \mathrm{~mm}$, which is generally regarded as the maximum thickness suitable for ${ }^{106} \mathrm{Ru}$ brachytherapy. Furthermore, it is expected that the radiation dose to the scleral surface will need to be reduced to decrease the risk of radiation-induced toxicities.

Choroidal melanomas are relatively resistant to radiation, and the brachytherapy dose needed to eradicate this tumor is associated with a considerable risk of radiation damage to ocular structures. In our analysis, the actuarial rate of radiation related side effects that require further treatment was $7 \%$. One of these patients underwent enucleation for persistent phthisis after brachytherapy. This patient received a high dose of radiation to the sclera exceeding 1,000 Gy with a high dose rate (dose rate to sclera, $502.9 \mathrm{cGy} / \mathrm{hr}$ ). Overall, $62 \%$ of patients who had effective visual function upon initial diagnosis eventually experienced complete loss of their visual acuity at their last follow-up, which is comparable to the results from other large series $[20,21]$. Half of these patients had a tumor close to the macula or the optic disc. Radiation induces occlusive vasculopathy with neovascularization and retinal edema, resulting in deterioration of visual acuity that continues for years after treatment. Increased doses and dose rates to the macula and the optic disc are associated with poorer visual outcomes [22].

According to our data, $80 \%$ of patients initially treated with brachytherapy could be spared from enucleation. As expected, the eye-preservation rate of the large group was lower than that of the small group (3-year eye-preservation rate, $70 \%$ vs. $94 \% ; \mathrm{p}=0.047$ ). Additionally, the overall outcome of patients in our large group was lower than that of Caucasian patients, who showed a 70\%-90\% eye-preservation rate after ${ }^{125} \mathrm{I}$ brachy-therapy for large melanomas and a $82 \%-88 \%$ rate after ${ }^{106} \mathrm{Ru}$ brachytherapy for any sized tumors. Although $70 \%$ of patients in the large group were rescued from enucleation, their oncologic outcome was still not satisfactory (3-year FFDM, 76\%; 3-year OS, 72\%). This could be explained by the distinct nature of the tumor in Asian 
patients [23].

For these patients, other therapeutic approaches, including proton therapy, which can access the full thickness of a large tumor, may improve local control $[24,25]$. However, an effective method for reducing metastasis is still lacking, and survival rates for these patients do not differ significantly between treatment methods, including brachytherapy, enucleation, and GKS $[1,13]$. Hawkins reported that no effective treatment for metastatic ocular melanoma has been found during the process of organizing Collaborative Ocular Melanoma Study trials [26]. However, a recent study reported the safety and efficacy of immuno-therapy with antiCTLA-4 antibody, an effective treatment for metastatic cutaneous melanoma, for treatment of metastatic uveal melanoma [27]. Based on the results of the present and previous studies, further investigation is warranted. Moreover, early detection of distant metastases at the time of diagnosis is essential to avoiding unnecessary local treatment and to evaluation of potential adjunctive treatments.

In interpreting our data, the limitations of retrospective analysis, including patients and treatment selection, must be considered. Although the absolute indication for brachyther- apy with additional local treatment is unclear, use of this treatment seems to reliably be recommended for the improvement of local control. Another limitation lies in the difficulty of evaluating toxicities, including determining whether changes in the retina were attributed to radiation alone or to tumor growth as well, and if these changes may have existed at the time of initial diagnosis. Toxicity analysis included only the patients requiring further treatment in this study; therefore, the incidence of toxicities cannot be applied directly to all patients with choroidal melanomas treated with ${ }^{106} \mathrm{Ru}$ brachytherapy. As the cohort is relatively small, there were limitations in conducting a complete statistical analysis. Additionally, it is necessary to verify the prognostic factors identified in our data. Nevertheless, the strengths of our study are that our patients received relatively homologous treatments based on their tumor size, and that they were performed in a single institution.

\section{Conflicts of Interest}

Conflict of interest relevant to this article was not reported.

\section{References}

1. Kwon HJ, Ko JS, Kim M, Lee CS, Lee SC. Prognosis of choroidal melanoma and the result of ruthenium brachytherapy combined with transpupillary thermotherapy in Korean patients. Br J Ophthalmol. 2013;97:653-8.

2. Diener-West M, Earle JD, Fine SL, Hawkins BS, Moy CS, Reynolds SM, et al. The COMS randomized trial of iodine 125 brachytherapy for choroidal melanoma, III: initial mortality findings. COMS Report No. 18. Arch Ophthalmol. 2001;119: 969-82.

3. Diener-West M, Earle JD, Fine SL, Hawkins BS, Moy CS, Reynolds SM, et al. The COMS randomized trial of iodine 125 brachytherapy for choroidal melanoma, II: characteristics of patients enrolled and not enrolled. COMS Report No. 17. Arch Ophthalmol. 2001;119:951-65.

4. Collaborative Ocular Melanoma Study Group. Ten-year follow-up of fellow eyes of patients enrolled in Collaborative Ocular Melanoma Study randomized trials: COMS report no. 22. Ophthalmology. 2004;111:966-76.

5. Lommatzsch PK, Werschnik C, Schuster E. Long-term followup of Ru-106/Rh-106 brachytherapy for posterior uveal melanoma. Graefes Arch Clin Exp Ophthalmol. 2000;238: 129-37.

6. Potter R, Janssen K, Prott FJ, Widder J, Haverkamp U, Busse $\mathrm{H}$, et al. Ruthenium-106 eye plaque brachytherapy in the conservative treatment of uveal melanoma: evaluation of 175 patients treated with 150 Gy from 1981-1989. Front Radiat
Ther Oncol. 1997;30:143-9.

7. Seregard S, aft Trampe E, Lax I, Kock E, Lundell G. Results following episcleral ruthenium plaque radiotherapy for posterior uveal melanoma. The Swedish experience. Acta Ophthalmol Scand. 1997;75:11-6.

8. Shields CL, Bianciotto C, Rudich D, Materin MA, Ganguly A, Shields JA. Regression of uveal melanoma after plaque radiotherapy and thermotherapy based on chromosome 3 status. Retina. 2008;28:1289-95.

9. Lommatzsch PK, Weise B, Ballin R. Optimization of irradiation time in the treatment of malignant melanoma of the choroid with beta applicators (106Ru/106Rh). Klin Monbl Augenheilkd. 1986;189:133-40.

10. Yarovoy AA, Magaramov DA, Bulgakova ES. The comparison of ruthenium brachytherapy and simultaneous transpupillary thermotherapy of choroidal melanoma with brachytherapy alone. Brachytherapy. 2012;11:224-9.

11. Shields CL, Cater J, Shields JA, Chao A, Krema H, Materin M, et al. Combined plaque radiotherapy and transpupillary thermotherapy for choroidal melanoma: tumor control and treatment complications in 270 consecutive patients. Arch Ophthalmol. 2002;120:933-40.

12. Lommatzsch PK. Results after beta-irradiation (106Ru/106Rh) of choroidal melanomas: 20 years' experience. Br J Ophthalmol. 1986;70:844-51.

13. Hawkins BS; Collaborative Ocular Melanoma Study Group. 
The Collaborative Ocular Melanoma Study (COMS) randomized trial of pre-enucleation radiation of large choroidal melanoma: IV. Ten-year mortality findings and prognostic factors. COMS report number 24. Am J Ophthalmol. 2004;138: 936-51.

14. Krema H, Heydarian M, Beiki-Ardakani A, Weisbrod D, Xu $\mathrm{W}$, Simpson ER, et al. A comparison between ${ }^{125}$ Iodine brachytherapy and stereotactic radiotherapy in the management of juxtapapillary choroidal melanoma. Br J Ophthalmol. 2013;97:327-32.

15. Kaiserman N, Kaiserman I, Hendler K, Frenkel S, Pe'er J. Ruthenium-106 plaque brachytherapy for thick posterior uveal melanomas. Br J Ophthalmol. 2009;93:1167-71.

16. Mashayekhi A, Shields CL, Rishi P, Atalay HT, Pellegrini M, McLaughlin JP, et al. Primary transpupillary thermotherapy for choroidal melanoma in 391 cases: importance of risk factors in tumor control. Ophthalmology. 2015;122:600-9.

17. Seregard S, Landau I. Transpupillary thermotherapy as an adjunct to ruthenium plaque radiotherapy for choroidal melanoma. Acta Ophthalmol Scand. 2001;79:19-22.

18. Kreusel KM, Bechrakis N, Riese J, Krause L, Wachtlin J, Foerster MH. Combined brachytherapy and transpupillary thermotherapy for large choroidal melanoma: tumor regression and early complications. Graefes Arch Clin Exp Ophthalmol. 2006;244:1575-80.

19. Augsburger JJ, Schneider S, Narayana A, Breneman JC, Aron BS, Barrett WL, et al. Plaque radiotherapy for choroidal and ciliochoroidal melanomas with limited nodular extrascleral extension. Can J Ophthalmol. 2004;39:380-7.
20. Damato B, Patel I, Campbell IR, Mayles HM, Errington RD. Visual acuity after Ruthenium ${ }^{106}$ brachytherapy of choro-idal melanomas. Int J Radiat Oncol Biol Phys. 2005;63:392-400.

21. Verschueren KM, Creutzberg CL, Schalij-Delfos NE, Ketelaars M, Klijsen FL, Haeseker BI, et al. Long-term outcomes of eyeconserving treatment with Ruthenium ${ }^{106}$ brachytherapy for choroidal melanoma. Radiother Oncol. 2010;95:332-8.

22. Lommatzsch PK, Alberti W, Lommatzsch R, Rohrwacher F. Radiation effects on the optic nerve observed after brachytherapy of choroidal melanomas with 106Ru/106Rh plaques. Graefes Arch Clin Exp Ophthalmol. 1994;232:482-7.

23. Sakamoto T, Sakamoto M, Yoshikawa H, Hata Y, Ishibashi T, Ohnishi $Y$, et al. Histologic findings and prognosis of uveal malignant melanoma in japanese patients. Am J Ophthalmol. 1996;121:276-83.

24. Bensoussan E, Thariat J, Maschi C, Delas J, Schouver ED, Herault $\mathrm{J}$, et al. Outcomes after proton beam therapy for large choroidal melanomas in 492 Patients. Am J Ophthalmol. 2016;165:78-87.

25. Konstantinidis L, Roberts D, Errington RD, Kacperek A, Heimann H, Damato B. Transpalpebral proton beam radiotherapy of choroidal melanoma. Br J Ophthalmol. 2015;99: 232-5.

26. Hawkins BS. Collaborative ocular melanoma study randomized trial of I-125 brachytherapy. Clin Trials. 2011;8:661-73.

27. Danielli R, Ridolfi R, Chiarion-Sileni V, Queirolo P, Testori A, Plummer R, et al. Ipilimumab in pretreated patients with metastatic uveal melanoma: safety and clinical efficacy. Cancer Immunol Immunother. 2012;61:41-8. 